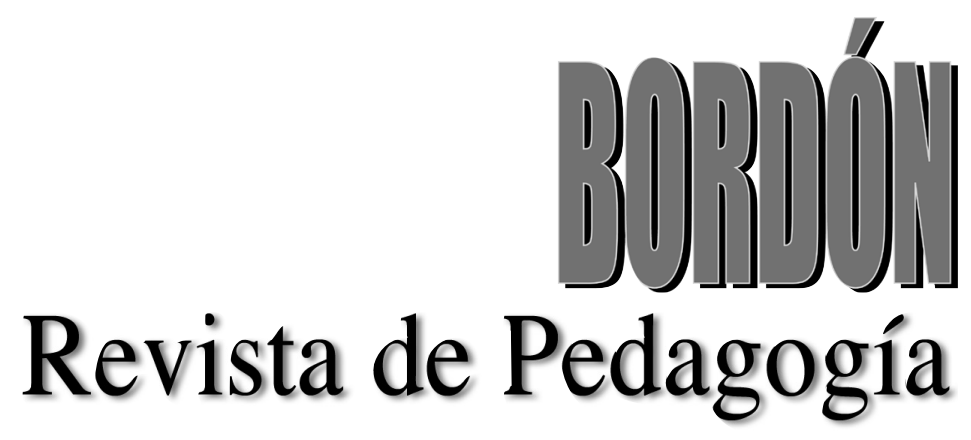

Volumen 65

Número 3

2013 



\title{
LA LABOR DEL MAESTRO EN LOS CUADERIOS ESCOLARES: UN ESTUDIO DE CASOS
}

\section{The teacher's labour in school exercise books: a case study}

\author{
CARMEN SANCHIDRIÁN BLANCO \\ Universidad de Málaga
}

BÁRBARA ARIAS GÓMEZ

Consejería de Educación de la Junta de Andalucía

INTRODUCCIÓN. Los cuadernos escolares son importantes fuentes documentales que posibilitan el estudio de la metodología, estrategias y patrones de enseñanza sobre los que cada maestro se apoyaba para enseñar. Constituyen un registro de su forma de trabajar en un espacio y tiempo concretos, en este caso, en la provincia de Málaga durante el nacionalcatolicismo. La investigación se centra en una colección de cuadernos escolares que se toman como fuentes primarias, enmarcados en un contexto histórico-educativo específico. MÉTODO. Se ha efectuado un estudio de carácter cualitativo, considerando categorías emergentes que han ido surgiendo en el proceso de revisión de los cuadernos. De esta manera se posibilitaba la inclusión de nuevas variables que podían proporcionarnos los documentos. No se trataba de partir de categorías conceptuales previas, sino de enunciarlas a medida que se revisaban los datos con el fin de realizar un análisis descriptivo. Ese análisis nos permite acceder al conocimiento de procesos subjetivos en los que nos interesa la perspectiva de los actores sociales intervinientes. RESULTADOS. Analizar los cuadernos nos ha permitido poner de manifiesto las potencialidades y limitaciones que poseen como fuente para la investigación en historia de la educación, así como deducir la organización de los tiempos escolares y la intervención del maestro en el proceso de evaluación. DISCUSIÓN. A partir de estos resultados, hemos podido concluir que los cuadernos escolares son eficaces instrumentos de evaluación que permiten a los docentes valorar de forma continua los procesos de enseñanza y orientar los aprendizajes posteriores. Además, ofrecen a los alumnos la posibilidad de integrar sus conocimientos de forma positiva y nos permiten recuperar los procesos de aprendizaje asociados a distintos modelos de escuela.

Palabras clave: Cuadernos escolares, Historia de la educación, Evaluación formativa.

\section{Introducción}

Descubrir la labor de un maestro a través de los cuadernos escolares nos va a permitir conocer de qué manera ordenaba, programaba y evaluaba la actividad de acuerdo con un modelo de escuela, así como evidenciar las experiencias escolares cotidianas que tuvieron lugar en el tiempo histórico que encierran y reproducir los tiempos vividos por el maestro y el alumno dentro del aula. Se trata de estudiar los contenidos de los cuadernos y de mostrar las estrategias de enseñanza asociadas a situaciones culturales concretas y delimitadas en un contexto específico. 
Estos cuadernos permiten observar la toma de decisiones intencionales y programadas que el maestro realizó en la elección de los contenidos que, de acuerdo con los recursos que poseía, le permitirían alcanzar los objetivos propuestos. En ellos ha quedado plasmada la mediación del maestro y parte de la recepción del alumno. Nos referimos a todo ello como un proceso que puede que no se complete, pues uno de los límites de los cuadernos es, precisamente, que no permiten conocer en qué medida han sido asimilados los conocimientos transmitidos ni su grado de aceptación o rechazo (Mahamud, 2010). No hay que olvidar que los cuadernos escolares poseen la cualidad de mostrar lo que verdaderamente se enseñaba en la escuela, a diferencia de otras fuentes como puedan ser los libros de texto (Badanelli y Mahamud, 2007); su estudio permite descubrir las diferencias entre el "conocimiento socialmente legitimado" (Apple, 1993: 109) que propone, en nuestro caso, la Enciclopedia Álvarez - manual que sirvió de apoyo al maestro-y los contenidos de los cuadernos, como reproducción de lo que el maestro enseñaba.

Los cuadernos escolares, como objetos escolares, son portadores de símbolos asociados al ámbito educativo predominantes en cada época: ideología, valores y religión (Del Pozo y Ramos, 2003). En esta línea, es necesario poner de manifiesto la importancia que poseen como fuente para la investigación en historia de la educación ya que son materialescargadosdeinformación relevantesobre el día a día escolar, el tiempo, el currículo implícito, la organización y la vida escolar de los centros.

Conviene resaltar que la evaluación fue una de las funciones más importantes que cumplieron los cuadernos escolares, entendiéndose esta como un proceso que permitía al maestro la recogida de información sobre las actividades de enseñanza, su análisis e interpretación y la posterior toma de decisiones (Rosales, 1990). La elaboración del cuaderno promovía el encuentro entre maestro y alumno y la estructuración del conocimiento, mostrando los avances y obstáculos del alumno pudiendo aprender con ellos de los errores propios y ajenos. Podemos descubrir en ellos una visión formativa de la evaluación, pues a través de la escritura se materializan y sintetizan ideas que sirven para la reflexión sobre los resultados alcanzados en el proceso de enseñanza aprendizaje y muestran la calidad de un proceso compartido entre alumno, maestro y familia (Shores, 2007). Los cuadernos escolares conciben el aprendizaje como un largo proceso a través del cual el alumno construye su conocimiento mediante el desarrollo de las actividades que el maestro propone (Jorba y Sanmartí, 2000).

\section{Método}

Este estudio de casos se lleva a cabo sobre seis cuadernos que datan de los años 1957, 1958 y 1959, analizando las siguientes cuestiones: la organización de los tiempos en la práctica escolar diaria; el papel del maestro como mediador en la transmisión de actitudes, conductas y valores, y su presencia en el proceso de evaluación y control.

El autor de los cuadernos es José García Carrión, alumno del Grupo Escolar "García del Olmo", la primera Escuela Graduada de niños de Cártama que se inauguró en el curso 1956-1957. Su maestro fue Francisco Segovia Ruiz, maestro nacional y representante del SEM. La escuela contaba con tres secciones. Antes de su incorporación al Grupo, el alumno había asistido a la Escuela Unitaria $n^{\circ} 1$ de Cártama, ubicada en la casa del maestro Francisco Romero Martín. El alumno tenía doce años cuando se incorporó al Grupo Escolar donde estudió durante tres años. $\mathrm{Su}$ trabajo quedó reflejado en los cuadernos CI, CIII, CIV, CV y CVI. El cuaderno CII es el producto final de las clases de apoyo recibidas en casa de un maestro que impartía "clases de pago", Antonio Vargas, a las que acudía por las tardes, sábados incluidos.

Los cuadernos son el objeto sobre el que vamos a trabajar. Se trata de conocer las formas de enseñar de la escuela del nacionalcatolicismo y de recuperar, a través de ellos, unas relaciones 
maestro-alumno que ofrecen una visión particular de lo que ocurrió en el único Grupo Escolar ubicado en la localidad rural de Cártama, durante el periodo que abarcan los cuadernos. El estudio se centra en un caso que presenta interés intrínseco para descubrir la realidad de una escuela en particular, por lo que la generalización no es un objetivo de la investigación (Stake, 1998). A partir del análisis y la descripción de estos documentos escritos, recuperamos las acciones que se llevaron a cabo en el desarrollo de la actividad escolar, manteniendo una estrecha relación con el tiempo puesto que, a partir de los cuadernos, podemos reconstruir el pasado de la escuela desde el presente.

En un primer análisis, observamos que los cuadernos presentan diferencias en cuanto a las materias estudiadas, la estructura, la organización de los tiempos en el aula y las señales y marcas empleadas para la corrección. Por ello, para llevar a cabo el estudio, hemos agrupado los cuadernos en tres categorías:

- Cuadernos CI, CIII, CIV y CVI. Pertenecen al mismo maestro y presentan la misma estructura y contenidos.

- Cuaderno CV. Pertenece al mismo maestro que los de la categoría anterior (y al mismo periodo que los cuadernos IV y VI), pero es un cuaderno de evaluación de las lecciones.

- Cuaderno CII. Pertenece al maestro de las clases de apoyo y presenta una estructura de contenidos diferente.
La distribución temporal de los cuadernos se detalla en la tabla 1.

\section{Objetivos}

El estudio de esta colección de cuadernos escolares nos permite analizar las producciones cotidianas que tuvieron lugar en el aula desde la perspectiva maestro-alumno, pudiéndose identificar con un contexto histórico concreto. El estudio se centra en encontrar la labor del maestro en los cuadernos, en descubrir su intervención en las correcciones que realiza sobre el cuaderno y su forma de enseñar. Por tanto, se pretende realizar un estudio sobre los cuadernos escolares con el fin de identificar en ellos los siguientes aspectos:

1. La labor desempeñada por el maestro en la actividad escolar: orden, evaluación, motivación.

2. Los contenidos que se trabajaban en la escuela, su organización y secuenciación.

3. Los aspectos personales y subjetivos de los actores que intervinieron en los cuadernos.

\section{Contexto histórico-educativo}

Durante la dictadura franquista, los instrumentos pedagógicos trataban de transmitir un sistema de valores basado en el tradicionalismo, autoridad, obediencia, orden y religión. Se idealizó un modelo de familia y de escuela (Mayordomo, 1997)

TABLA 1. Distribución temporal de los cuadernos

\begin{tabular}{lcccccc} 
Cuadernos & $\begin{array}{c}\text { Cuaderno I } \\
(\text { CI })\end{array}$ & $\begin{array}{c}\text { Cuaderno II } \\
(\text { CII })\end{array}$ & $\begin{array}{c}\text { Cuaderno III } \\
(\text { CIII })\end{array}$ & $\begin{array}{c}\text { Cuaderno IV } \\
(\text { CIV })\end{array}$ & $\begin{array}{c}\text { Cuaderno V } \\
(\text { CV })\end{array}$ & $\begin{array}{c}\text { Cuaderno VI } \\
(\text { CVI })\end{array}$ \\
\hline Curso & $1956-1957$ & $1957-1958$ & $\begin{array}{c}1957-1958 \\
1958-1959\end{array}$ & $1958-1959$ & $1958-1959$ & $1958-1959$ \\
& & & $12 / 04 / 1958$ & $26 / 01 / 1959$ & $23 / 01 / 1959$ & $05 / 05 / 1959$ \\
\hline Fomienzo & $12 / 01 / 1957$ & $10 / 01 / 1958$ & $15 / 07 / 1959$ & $06 / 06 / 1959$ \\
\hline
\end{tabular}


y la educación fue un instrumento de fuerte contenido político e ideológico poniéndose las escuelas al servicio de la ideología del Estado. Se trataba de un modelo de escuela caracterizado por la confesionalidad del Estado y por la absoluta presencia de los principios que regían en el nacionalcatolicismo (Escolano, 2002).

La escuela rural era pobre en cantidad y calidad $y$, dado que se consideraba aceptable que hubiera un buen porcentaje de población analfabeta (Navarro, 1990), los esfuerzos para redimirla de esa pobreza fueron escasos. Además, la tasa de analfabetismo en la provincia de Málaga, en 1950, era del 34\%, el doble de la nacional. En este sentido, corresponde distinguir dos acepciones de la escuela rural en España durante estos años (García, 1969: 367), una de tipo administrativo, que es la que contenían implícitamente la Ley de Educación Primaria de 1945 y el Estatuto del Magisterio Nacional Primario de 24 de octubre de 1947, y otra que justificaba la situación de inferioridad en que se encontraba esta escuela con una población predominantemente campesina y un ambiente denso de incultura que hacían de esta una escuela "opuesta" a la urbana (Serrano de Haro, 1941).

El maestro de esta época se debía caracterizar por ser un buen funcionario, cumplidor y buen cristiano, obligado a asistir a misa con sus alumnos. Para Serrano de Haro (1944), el maestro que formara parte de la escuela rural debía hacerlo por vocación. Su labor se controlaba directamente por la Inspección de Enseñanza Primaria y, de manera indirecta, por los Cuestionarios Nacionales, a los cuales debían ajustarse los programas, los libros de texto y los de lectura. Es obvio que figuras como el cura o el alcalde, y podríamos decir que todo el pueblo, también controlaban la labor de estos maestros. Los contenidos de los cuadernos escolares reflejan todo esto pues poseen la capacidad de registro de los valores y actitudes que el maestro inculcaba (Del Pozo y Ramos, 2001). Igualmente, son considerados portadores de huellas que mantienen una correspondencia con ideologías, valores y prácticas pedagógicas (Sacchetto, 1986).

\section{El maestro y la organización de la jornada escolar}

Los cuadernos de clase fueron, en las escuelas primarias de esta época, uno de los medios didácticos en los que se centró la actividad escolar. Consideramos los cuadernos escolares como medios didácticos porque son recursos preparados con la intención de facilitar el proceso de aprendizaje. El maestro los utilizaba para transmitir conceptos y valores y para desarrollar en el alumno actitudes como el orden, la pulcritud y la buena escritura. De hecho, la pulcritud y minuciosidad de muchos de los cuadernos encontrados son destacadas frecuentemente en las investigaciones sobre los mismos (Badanelli y Mahamud, 2011: 98). No se nos escapa que precisamente esta característica ha podido contribuir a la conservación de estos, mientras que otros elaborados con menos cuidado no han sido conservados.

Entre los elementos comunes que componen los cuadernos escolares como medios didácticos, destacamos la lección (unidad básica de información sobre la que se centra la jornada escolar), los códigos icónicos (se utilizan las imágenes como representaciones intuitivas que se acercan a la realidad), diversidad de señalizaciones (rótulos, subrayados, distintos tipos de letra y utilización de los colores) y los resultados de aprendizaje (quedaron como producto final plasmados en el cuaderno, ofreciendo la posibilidad de poder ser medidos).

Con la utilización del cuaderno, el maestro intentaba que el alumno se apropiara de los contenidos expuestos en clase. De esta manera, el alumno debía integrar la información que se le proporcionaba y estructurar los conocimientos dotándolos de sentido.

Los cuadernos escolares guiaron el aprendizaje, motivaron y potenciaron el desarrollo de un 
conjunto de habilidades entre las que distinguimos:

- La observación, la interpretación y la representación. Debían adquirirse al copiar de la pizarra los contenidos que el maestro exponía en cada lección o reproducía textos de la Enciclopedia. Se conseguía así que el alumno atendiese, se fijara y aprendiese a concentrarse.

- La organización de la información. En el cuaderno destacan los elementos básicos de la lección como unidad de información. En este sentido, el alumno a través del cuaderno resaltaba y distinguía entre un tipo de contenido u otro, ya fueran lecciones, actividades, dictados, copiados, redacciones, consignas... Aprendía a ordenar, puesto que organizaba de forma sistemática la jornada escolar. El maestro seguía un orden que le servía de rutina.

- El desarrollo de la memoria. Los cuadernos permitían que el niño fuese capaz de codificar, conservar, evocar y recordar lo acontecido en clase.

- La evaluación. El alumno valoraba y juzgaba el resultado de su trabajo diario y podía compararlo con el de otros alumnos de la clase, además de conocer la valoración que el maestro confería a sus tareas.

Por el análisis de los cuadernos y las entrevistas realizadas al alumno, sabemos que el maestro utilizó como texto para el desarrollo de sus clases la Enciclopedia Álvarez de Tercer Grado. Las pautas metodológicas que hemos seguido para llegar a esta conclusión se basan en la utilización de una serie de indicadores, tales como las huellas de uso y marcas de fechas realizadas en el libro que utilizó el alumno —del que disponemos- y que se corresponden con el cuaderno, así como los vínculos que se pueden encontrar entre el libro y su reflejo en el cuaderno, justificados por la fidelidad entre los textos reproducidos por el alumno y los que propone el manual. Lo que preocupa en este modelo de enseñanza es la acumulación de información, que queda perfectamente reflejada en los cuadernos. Se presentan los contenidos como resultados, con un marcado carácter estático y permanente.

Era una forma de enseñar que enfatizaba los contenidos y la persona que los transmitía, por lo que la figura del maestro adquiere un papel relevante en este proceso. El maestro era el único poseedor del conocimiento, con capacidad de síntesis, puesto que reunía las distintas disciplinas, elementos separados, y las componía como un todo conectado, dándoles su propia orientación (Woods, 1989). Lo que el maestro tenía que enseñar ya estaba organizado. Lo prioritario eran los contenidos frente a los procedimientos de aprendizaje, que respondían a una fuerte sistematización y orden en su exposición. Toda la práctica escolar convergía en la lección, que era la unidad y el núcleo del currículo (Benso, 2006). De acuerdo con esto, podríamos recordar que la utilización de distintos medios didácticos da lugar a diferentes contenidos de aprendizaje y estructuras de la actividad (Rosales, 1990).

Otro aspecto observable a través de los cuadernos es la organización de los tiempos. Los cuadernos muestran el reparto de las actividades en unidades temporales, la distribución y el empleo diario del tiempo escolar (Viñao, 1998). El cuaderno es un reflejo del orden temporal en el que se van desarrollando las tareas, puesto que los trabajos se presentan de forma sucesiva; de esta manera lo escrito en la segunda página es temporalmente posterior a lo escrito en la primera. Además favorece el registro y reproducción de la práctica discursiva en el aula, ya que no permite la posibilidad de incorporar hojas sueltas. Tampoco el alumno podrá eliminar párrafos pues siempre escribe con tinta y la actividad de borrar dejaría huellas. Así, se constata que la secuencia espacial se identifica con la temporal (Gvirtz, 1999). 
La secuenciación de la jornada escolar correspondiente a los cuadernos agrupados en la primera categoría (CI, CIII, CIV y CVI), tal como la presentamos (gráfico 1), se ha obtenido a partir de las frecuencias de aparición de las distintas materias estudiadas cada día. Se observa que el maestro del Grupo Escolar organizaba la jornada comenzando con una lección de las distintas materias que debía tratar, y que combinaba a lo largo de la semana, para dar paso a un dictado, que suponía el refuerzo de los contenidos tratados en ella. Posteriormente, se realizaban problemas, combinando cálculo con ejercicios de matemáticas y finalizaba con lecciones de materias que requerían una menor concentración y esfuerzo intelectual para el alumno (formación religiosa, social, política o historia sagrada). En el gráfico 1, cada lección o actividad ocupa una superficie de tamaño irregular. Con ello, pretendemos indicar la frecuencia de aparición de estas según el día de la semana y no la duración o el tiempo dedicado dentro de la jornada. Los cuadernos nos van a permitir conocer el orden en que se impartieron las materias y si se estudiaron o no en los diferentes días de la semana, sin embargo, no nos resulta posible identificar la producción gráfica del alumno con el tiempo que el maestro le dedicó.
Así vemos cómo el maestro programaba la secuenciación de las áreas de estudio en función de su grado de dificultad, según el momento del día o de la semana, ponderando la disposición de las materias objeto de estudio. No hay que olvidar que se va a encontrar con dos dificultades: la presencia en el aula de diversos ritmos de aprendizaje y la asistencia intermitente del alumnado, ya que, en numerosas ocasiones, tenían que faltar a la escuela y dedicarse a las labores del campo para ayudar a sus familias. A pesar de la variedad de actividades y diversidad en cuanto a la utilización de los tiempos en la escuela, el maestro dotaba de gran uniformidad este proceso, en el que las tareas se presentaban con cierto aire de ritual y con un fuerte componente cíclico. En efecto, vemos cómo el contenido del trabajo va cambiando cada día, ofreciendo gran variedad a lo largo de la semana, pero siempre dentro de la continuidad (Jackson, 1975). Cada día, sin excepción, comenzaba la clase con el inicio de una nueva actividad. El maestro controlaba el tiempo que destinaba a la jornada escolar, pues no se encuentran actividades inconclusas en los cuadernos, lo que parece implicar que tenía programado su trabajo.

\section{GráfICo 1. Organización de las jornadas escolares. Cuadernos CI, CIII, CIV y CVI}
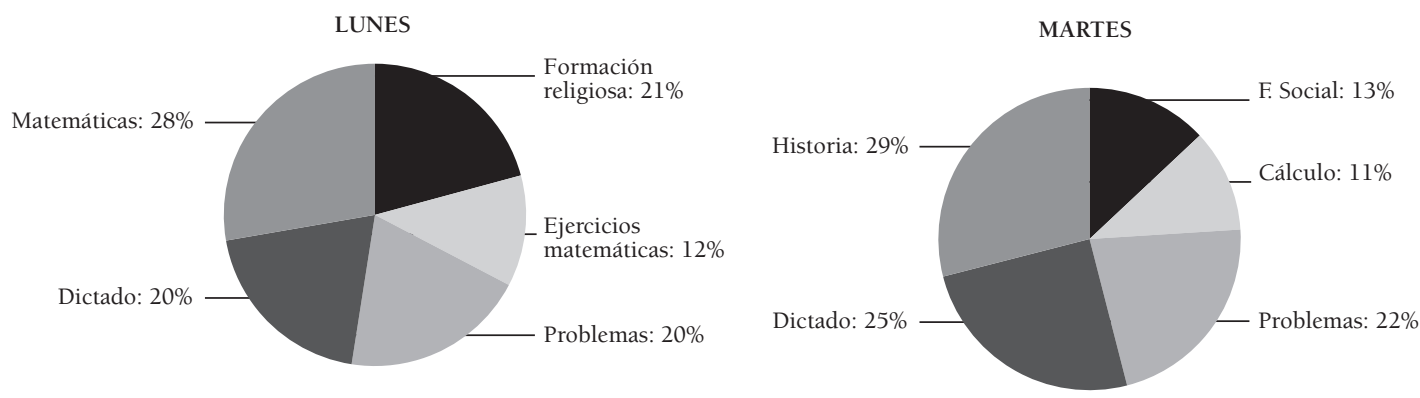


\section{GrÁfICo 1. Organización de las jornadas escolares. Cuadernos CI, CIII, CIV y CVI (cont.)}
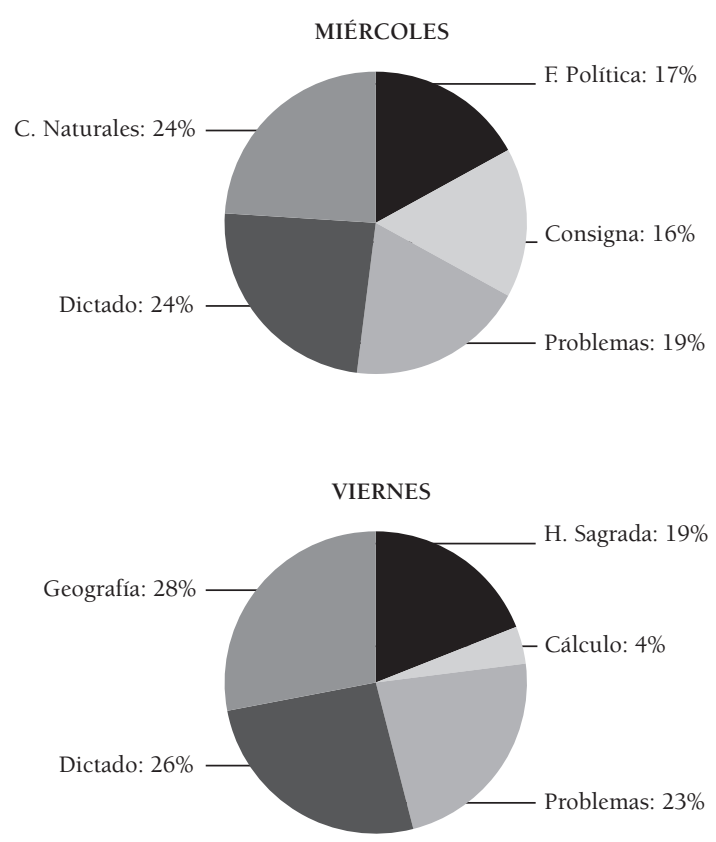

Los textos que el alumno reprodujo en sus cuadernos presentan una gran coincidencia con los que propone la Enciclopedia que el maestro tomaba como manual de referencia. No obstante, se aprecia la labor de mediador del maestro facilitando la compresión de los contenidos puesto que, en ocasiones, los textos se encuentran abreviados, las frases se simplifican y se obvian otros contenidos de ampliación que propone la Enciclopedia y que pudieran resultar complicados.

El maestro actuó como transmisor de la ideología dominante, en sus lecciones y a lo largo de la jornada escolar, moldeando la conciencia moral de sus alumnos e inspirándolos en el desarrollo de los valores e ideología que se esperaba que fueran adquiriendo en la escuela. "La escuela, pues, fue lugar de enseñanza, de adoctrinamiento, de vivencias religiosas, de catequesis, de seguimiento del año litúrgico" (Gervilla, 2006: 542). Es así cómo los cuadernos revelan
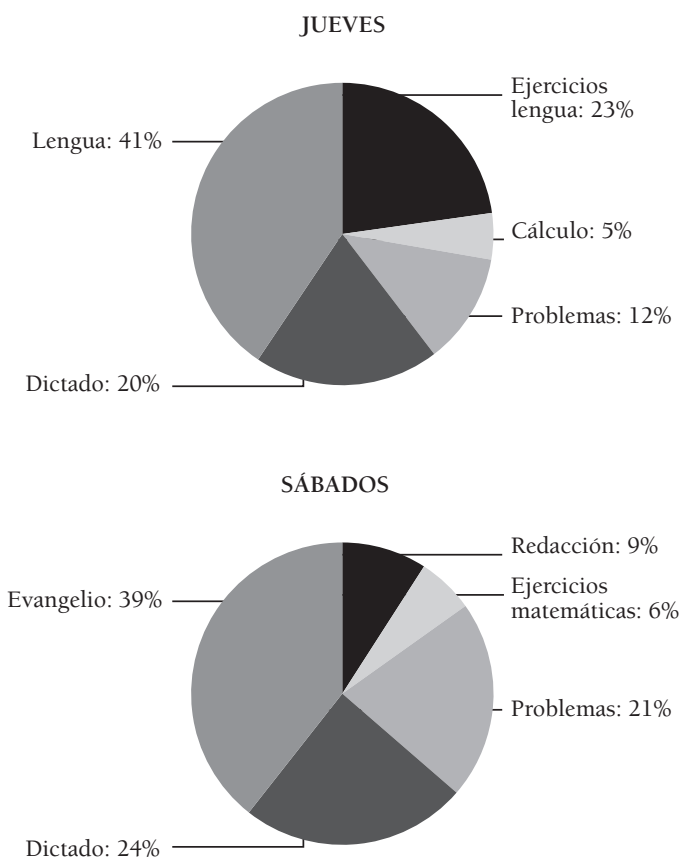

que la escuela es ese lugar donde se enseñaba el conocimiento legítimo y el conocimiento esperado, por ejemplo, la obediencia a la autoridad y el respeto a las reglas del juego (Lomas, 2002).

Otro aspecto que llama la atención es el grado de elaboración y la calidad que caracterizan a los cuadernos pudiendo verse, de nuevo, cómo el maestro conseguía desarrollar en el niño actitudes y destrezas como el hábito de limpieza, el orden, el esfuerzo y la dedicación, además del sentido estético, constituyendo los cuadernos una evidencia de la labor docente del maestro y de las dimensiones procedimentales y actitudinales del proceso de enseñanza-aprendizaje (Mahamud, 2010).

Analizando el cuaderno CII que el alumno elaboró en las clases particulares, observamos que este maestro tenía otra forma de enseñar, con una metodología muy distinta a la utilizada por 
IluSTRaCión 1. Cuaderno VI (1959) y Enciclopedia Álvarez, Tercer Grado (lecciones 33 y 34)
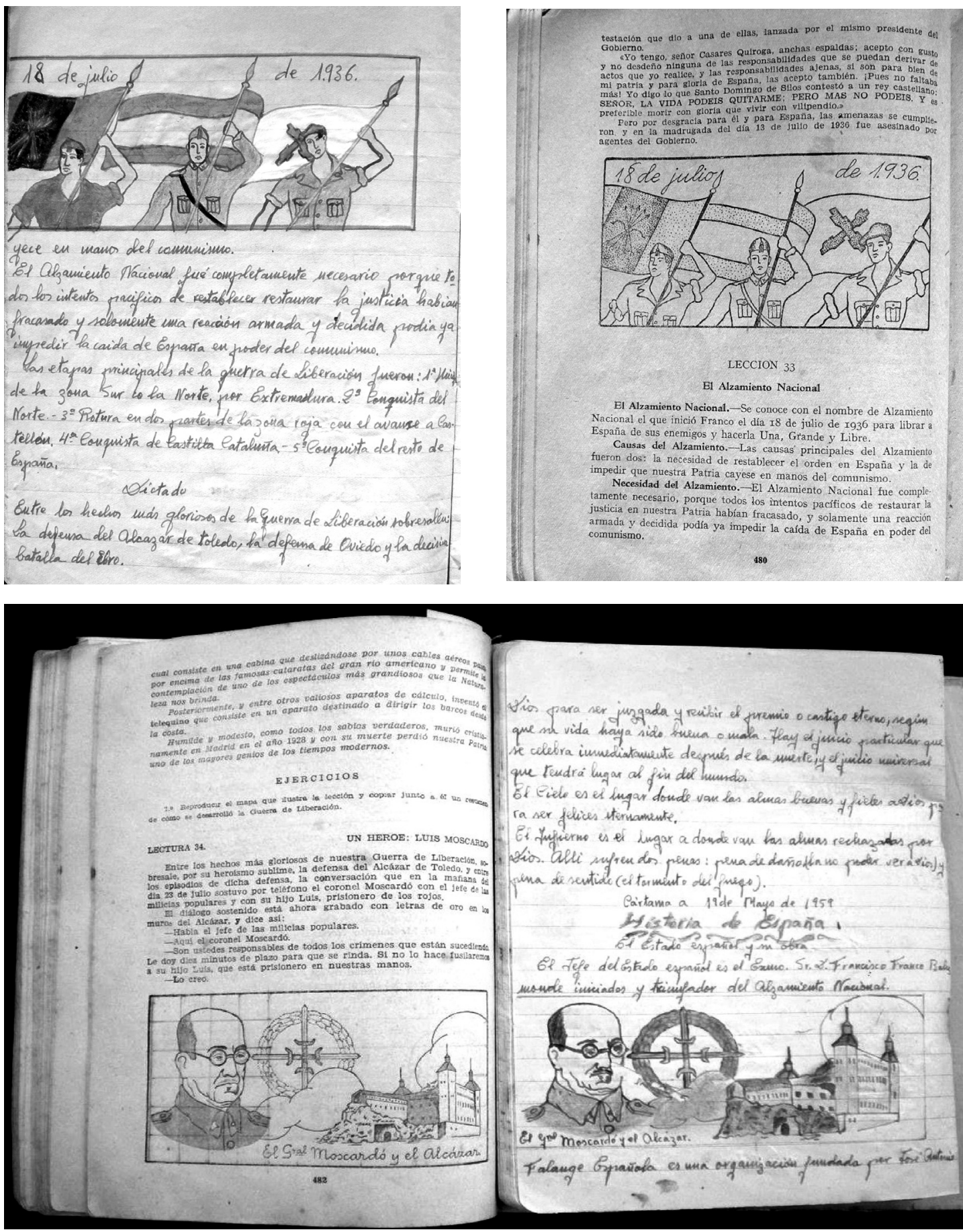
el maestro del Grupo Escolar. Posiblemente esto se debiera a que las enseñanzas del maestro de las "clases de pago" no estaban tan controladas oficialmente como las del maestro del Grupo Escolar, a que no disponía del mismo tiempo para impartir sus enseñanzas y a tener distintos objetivos. Sin embargo, ambos maestros siguen unas etapas determinadas: presentan unos contenidos limitados que permiten instruir al alumno, formulan una cuestión o problema sobre la materia expuesta y a través de la corrección conocen los resultados de forma inmediata (Gronlund, 1973).
El maestro que imparte las clases de apoyo comienza con un dictado, a continuación realiza un ejercicio de análisis gramatical, una actividad que llama copiado y, por último, el alumno debe copiar al menos diez veces las palabras que hubiera escrito con errores ortográficos en el dictado, bajo el título "faltas". Se observa que este tipo de tareas no se realizan en el trabajo propuesto por el maestro del Grupo Escolar y la mayor parte de la jornada escolar se dedica a la realización de ejercicios prácticos y de aplicación.

\section{Gráfico 2. Organización de las jornadas escolares. Cuaderno CII}

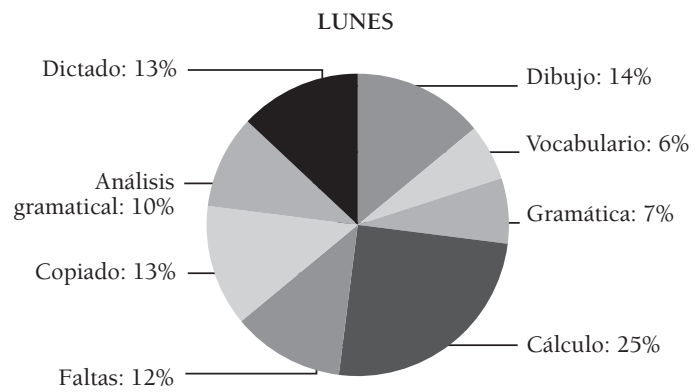

MIÉRCOLES

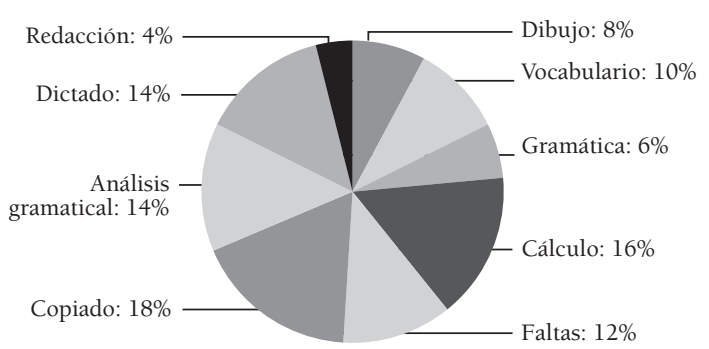

VIERNES

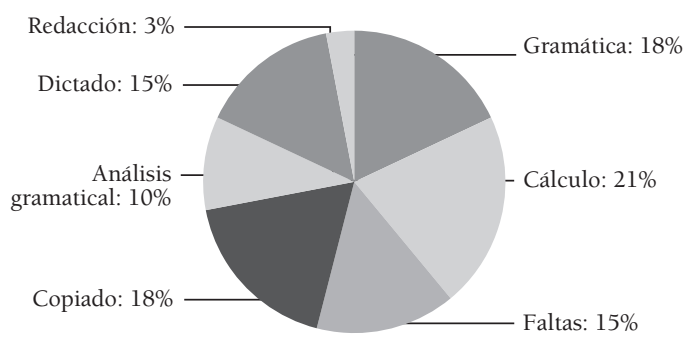

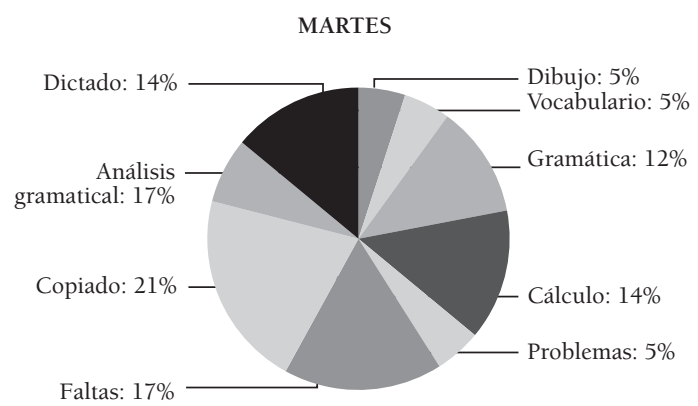

JUEVES

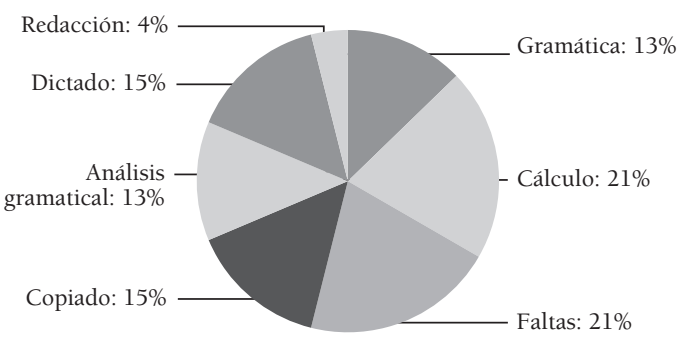

SÁBADOS

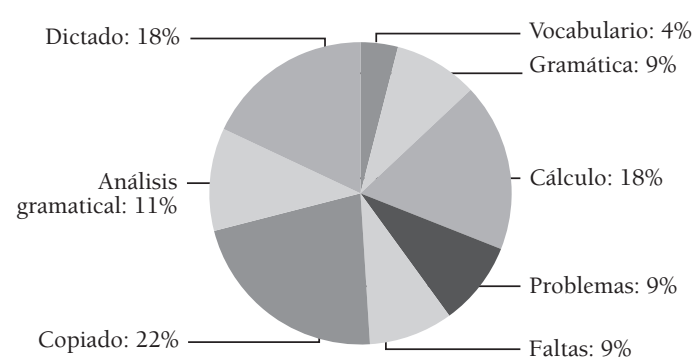


Los cuadernos reflejan, igualmente, momentos de complicidad con la clase a través de la realización de dictados y redacciones. Un ejemplo de ello, lo encontramos en un dictado de chistes escrito en el cuaderno CII, lo que da pie a suponer que el maestro tiene perfectamente controlado al grupo y se atreve a llevar a cabo este tipo de actividades:

"Dictado

Chistes

-Oye, Pepín, ¿cómo sacarías un gato de un pozo? -le pregunta Cristóbal a su hermanito.

-Pues con una cuerda.

_ ¡Anda ya tonto! Lo sacarías mojado."

En algunos ejercicios de redacción, el maestro propone temas con el fin de lograr un mayor acercamiento a la vida cotidiana del alumno y de esta manera compartir con él momentos vividos fuera del entorno escolar, potenciando así la complicidad en la relación alumnomaestro. Algunas de estas redacciones son "¿Qué hiciste ayer" o "¿Qué has hecho hoy?”.
De esta manera el maestro comparte las emociones del alumno y se establece una complicidad entre ellos. Así, en la primera redacción, el alumno describe con fervor la subida a la Ermita de la Virgen de los Remedios y deja constancia de la ilusión que le provoca la llegada al pueblo. En la segunda, "¿Qué has hecho hoy?", relata el acontecimiento. Es así cómo el maestro puede comprobar, además, que los hábitos y valores transmitidos en la escuela han sido adquiridos por el alumno.

\section{Los maestros y la evaluación de los cuadernos}

Cuando el maestro evaluaba la tarea cotidiana estaba reconociendo el esfuerzo realizado por el alumno, que podía apreciar con mucha claridad cómo se podía hacer mejor y aprender de ello. Y puesto que el cuaderno era un dispositivo de trabajo del alumno, como instrumento para el estudio, tenía que permitirle aprender de él. Siguiendo a Álvarez

IluSTRaCión 2. Dos maestros y dos tipos de correcciones: Cuadernos CV y CII
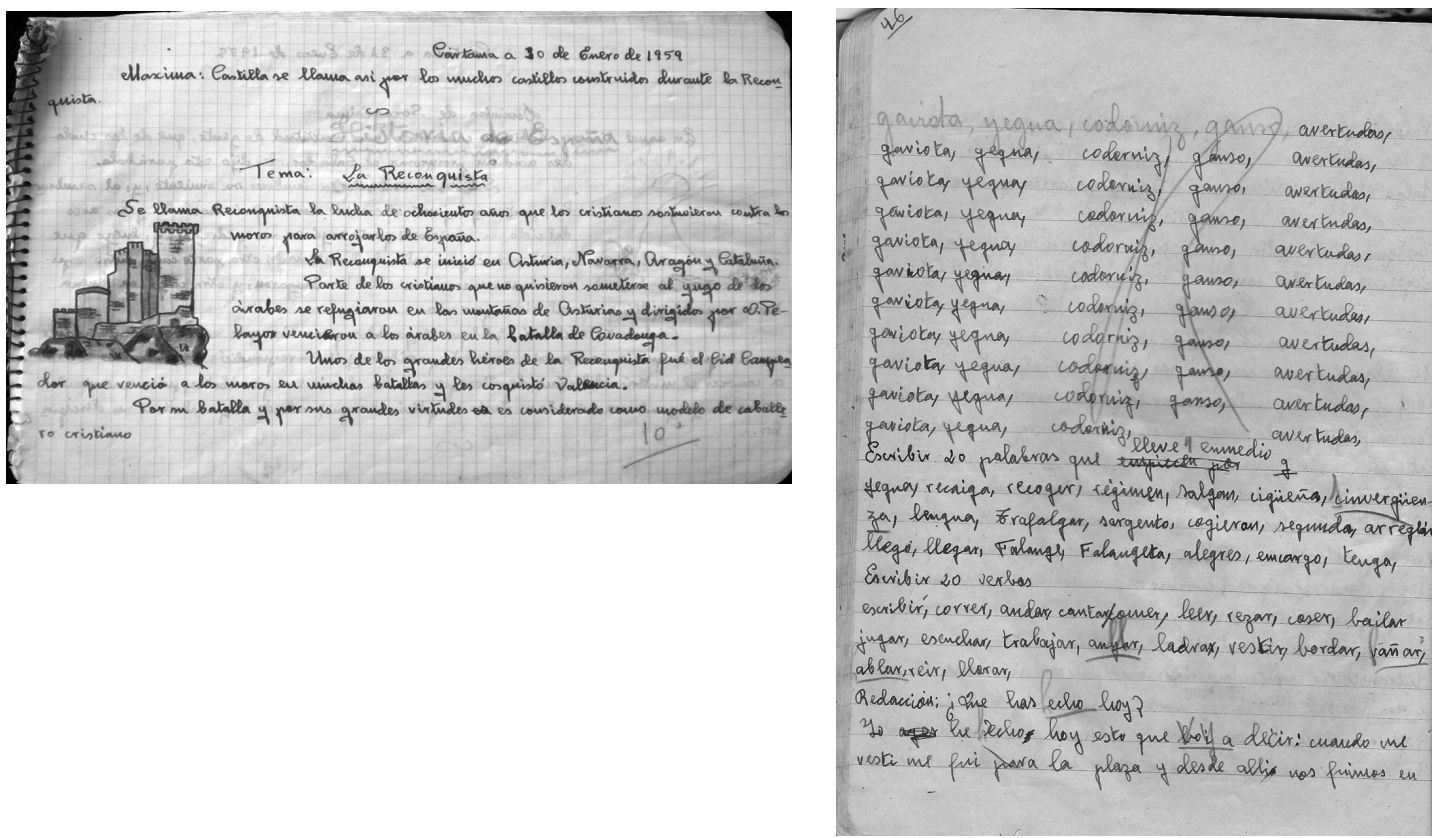
Méndez (1993), la evaluación es parte de la enseñanza y a medida que el alumno aprende, de forma simultánea, evalúa, razona, fundamenta... Es una actividad continuamente formativa que tiene su reflejo en los cuadernos y que forma parte del proceso educativo de este alumno.

Para estudiar las correcciones del maestro reflejadas en esta colección de cuadernos, hemos considerado la siguiente clasificación (Castillo y Sierra, 2008): correcciones ortográficas, calificaciones y correcciones de añadido de información.

En los cuadernos que el alumno elaboró en el Grupo Escolar encontramos que la evaluación del maestro se llevaba a cabo mediante códigos o signos compartidos cargados de significado para ambos. Por un lado, informaban del grado de consecución de los objetivos y, por otro, se marcaban los errores sin perder de vista que las correcciones servirían también al maestro para motivar en todo el proceso de aprendizaje al niño, puesto que las marcas y correcciones que descubrimos en los cuadernos no son en absoluto agresivas, están lejos de ser lesivas e incluso se podrían calificar de tímidas debido a su pequeño tamaño, pasando casi desapercibidas.

El maestro del Grupo Escolar evaluó los cuadernos sin apenas dejar marcas, ya que corregía incorporando pequeñas señales, casi siempre de color rojo, muy poco llamativas. En la mayoría de los casos marcó los errores de acentuación cometidos evitando el carácter sancionador de unas señales grandes o tachaduras sobre la producción escrita del alumno. Poseía una función instructiva, con la que se buscaba el desarrollo de aptitudes y hábitos de trabajo (Rosales, 1988). Se trataba de una evaluación que buscaba el estímulo del alumno, transmitir responsabilidad por el trabajo bien hecho y servir de refuerzo positivo considerando valioso el trabajo realizado. Y es que la forma en que el maestro informa al alumno de sus resultados y de la evolución de su aprendizaje, influye en la imagen que este se construye de sí mismo y condiciona sus posibilidades futuras (Parcerisa, 1996).

Por el contrario, el maestro de las clases de apoyo intervino en el cuaderno CII con correcciones de añadido de información. Con ello mostraba el resultado alcanzado y el que se debía tratar de alcanzar; utilizaba el lápiz, pero lo hacía con grandes marcas sobre el trabajo del niño, tratándose de una evaluación efectiva, con un carácter más sancionador que constructivo.

\section{Valores, conducta y actitudes transmitidos por los maestros}

La identificación del contenido de los valores tratados se hace a partir de esta clasificación:

- Valores individuales: "trabajo ordenado", "conciencia de uno mismo", "mentira", "trabajo bien hecho", "la conciencia", "nobleza de carácter", "higiene", "el esfuerzo"...

- Valores sociales: "forma de comportarse en la calle", "el buen hablar", "la amistad", "cortesía en familia", "deberes de padres e hijos", "en la escuela"...

- Valores patrióticos: "honor a la patria", "la unidad, la fuerza y el afán de grandeza de España", "el espíritu de lucha", "las ideas de juventud, guerra, muerte y victoria"...

Para poner de manifiesto el papel que en cada caso ocupa el maestro en la transmisión de valores, se estudia el tiempo que destina en sus clases al tratamiento de estos y de qué manera los trabaja. En primer lugar, se realiza un análisis de cada uno de los cuadernos por separado con el fin de determinar la frecuencia de aparición de cada clase de valor que el maestro trata y que permitirá deducir la importancia concedida a cada uno de ellos. 
Gráfico 3. Valores tratados en cada cuaderno (\%)

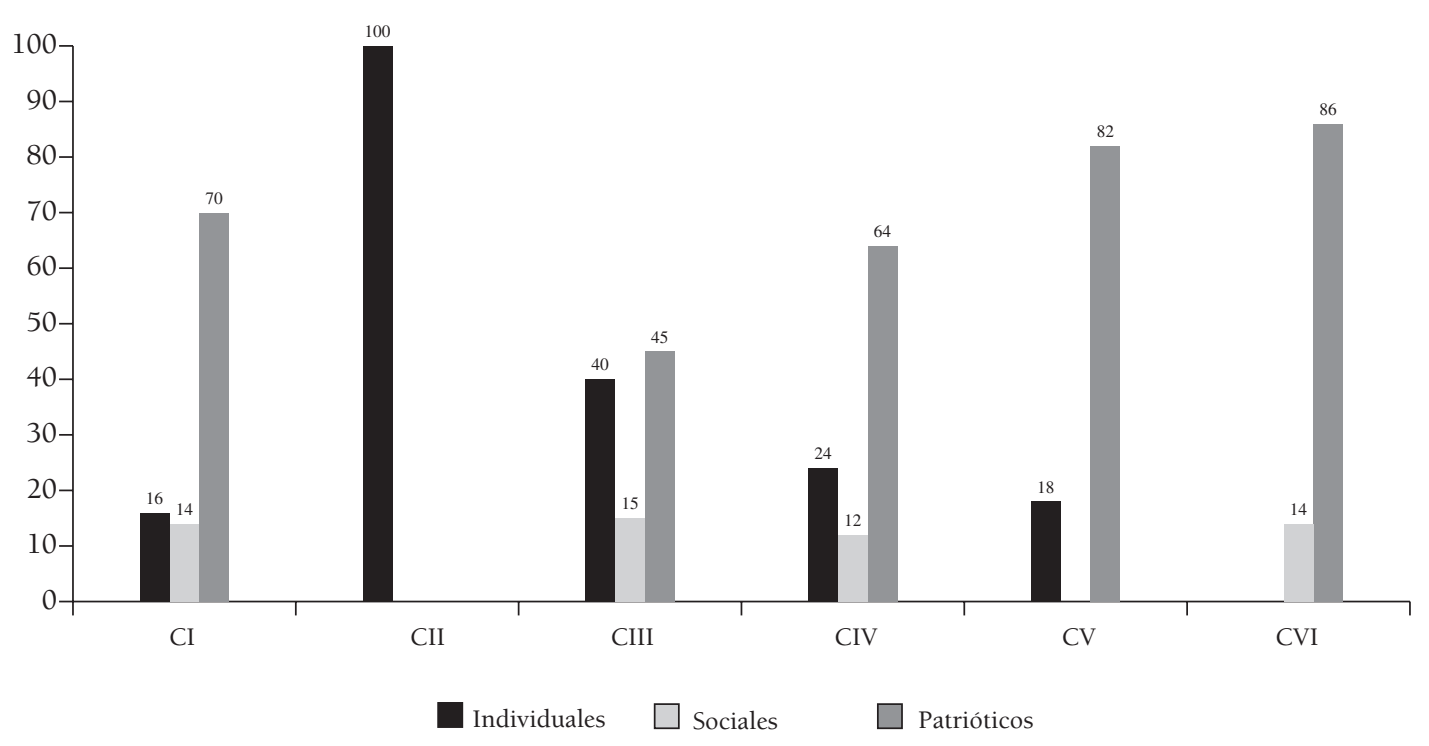

Con estos datos, se detecta que en los cuadernos CI, CIII, CIV y CVI, los del maestro del Grupo Escolar, se trabajan los tres tipos de valores, aunque predomina como mayor valor tratado el patriótico. No ocurre lo mismo en los cuadernos CII y CV.

El maestro de las clases de apoyo, solamente dedica dos ocasiones a valores individuales, aprovechando una de ellas para dejar entrever el papel que ocupa la mujer en la sociedad con un dictado titulado "Las ánimas":

\section{"Dictado \\ Las ánimas}

Había una vez una vieja que tenía una sobrina que había criado sujeta como cerrojo, y era muy buena niña, muy cristiana, pero encogida y poquita cosa. Lo que sentía la vieja era lo que iba a ser de su sobrina y así no hacía otra cosa que pedirle a Dios que le deparase un buen novio. Hacía los mandados en casa de una comadre suya pupilera."

En el cuaderno de evaluaciones, CV, el maestro utiliza como recurso la máxima para transmitir mensajes de valores individuales y trata los valores patrióticos mediante consignas.
Atendiendo al conjunto de los cuadernos agrupados en la primera categoría, CI, CIII, CIV y CVI, se observa que en todos ellos el maestro considera todos los valores que se deben tratar a excepción del cuaderno CVI en el que no trabaja los valores individuales. Destaca la importancia que concede a los patrióticos por lo que podemos deducir que el maestro se ciñe a las enseñanzas oficiales, dedicándose en menos ocasiones a la transmisión de los valores individuales.

De los datos que proporciona el cuaderno CII, de las clases particulares, se desprende que el maestro solamente trabaja los valores individuales y lo hace en dos ocasiones, ya que sus clases no se ajustan tanto al currículo oficial pues tienen la finalidad de reforzar lo aprendido por el alumno en la escuela y considera más importante otro tipo de lecciones. En ningún caso dedica tiempo a la transmisión de valores patrióticos ni sociales.

En el caso del cuaderno de evaluaciones, CV, se aprecia que las lecciones son más cortas; el maestro las denomina lecciones de Historia de España, Geografía, Formación Política, Historia 
Sagrada, Santo Evangelio, Ciencias y Lengua y comienza cada una con una "máxima". Lo más relevante de este cuaderno es la importancia que el maestro, al evaluar, confiere a los valores patrióticos frente a los individuales y la ausencia de los sociales.

Una de las funciones que cumple el cuaderno es la capacidad que posee como vehículo para fomentar los valores y actitudes que el maestro quiere inculcar. Así, en la lección $n^{\circ}$ 4 de Formación Familiar y Social de la Enciclopedia, se trata a través de un dictado el valor de la amistad. El maestro realiza una peculiar composición incorporando mensajes como "elegir bien nuestras compañías", que no aparecen en el texto original de la Enciclopedia. Pero además es aquí donde se aprecia su intervención puesto que, a modo de resumen y como mensaje final, utiliza la siguiente expresión: "y no olvidar la gran verdad del refrán que dice: Dime con quién andas y te diré quién eres".

Para la transmisión de valores a través de los dictados también se apoya en otros recursos didácticos que le permiten desarrollar sus enseñanzas. Es el caso de un dictado de "producción propia", tras el que los alumnos copian la fábula que utiliza para explicar el "trabajo bien hecho" en el cuaderno CI. Para su elaboración se basa en la "Fábula del gusano de seda y la araña”, de Tomás de Iriarte (1893: 16):

\section{"Dictado}

Siempre que os pongáis a hacer algún trabajo procurad hacerlo bien. Una vez hecho, nadie se preocupa de averiguar si tardasteis mucho o poco en hacerlo. Solo dirán: está bien o está mal. Nunca os parezcáis a la araña de la fábula."

\section{Conclusiones}

Es evidente el potencial que los cuadernos escolares ofrecen para investigar sobre los modelos escolares del pasado. Tomarlos como fuentes primarias nos ha permitido recuperar la organización de los contenidos y las formas de enseñar de un maestro en la escuela del nacionalcatolicismo. Pero además los cuadernos guardan unos silencios capaces de reflejar la forma en que el maestro ejerció la autoridad dentro del aula e incluso la complicidad en las relaciones entre maestro y alumno.

Los cuadernos escolares posibilitaron al maestro la evaluación interna ya que a través de ellos llegaba a conocer los progresos del alumno a lo largo del curso y detectaba sus dificultades. Constituyeron también un mecanismo de control, pues le permitían comprobar el grado de eficacia de los métodos elegidos para el desarrollo de sus enseñanzas. En esta época contribuyeron, además, a la evaluación externa, como testimonios del currículo enseñado ante las visitas de la Inspección.

Los contenidos de los cuadernos se presentan organizados en secuencias didácticas, como conjunto de actividades muy ordenadas, bien estructuradas y articuladas que perseguían la consecución de unos objetivos educativos concretos, con un principio y un final bien conocidos por el maestro. Se trataba de un método de carácter expositivo basado en la lección magistral, complementado con actividades de repetición sobre lo enseñado y siendo la Enciclopedia el texto utilizado como recurso fundamental. Observamos que el maestro consideraba la práctica como eje sobre el que formular la adquisición de procedimientos por lo que planteaba numerosos problemas con enunciados muy próximos a situaciones en las que los alumnos se podrían encontrar en la vida real. La secuencia de cada clase podría concretarse en: exposición, ejercicios guiados y prácticas autónomas. Además, hemos comprobado cómo integraba la evaluación en el proceso de formación, que el maestro completaba rescatando los valores culturales del contexto de la escuela, utilizándolos como elementos motivadores que contribuyeran al estímulo del proceso de aprendizaje del niño. 
En este sentido podemos destacar que la forma de enseñar del maestro se caracterizó por ser una enseñanza unidireccional (el maestro explicaba los procedimientos, su utilidad y los errores que aparecían en su ejecución), con imitación de modelos considerando las actitudes, normas y valores como contenidos que se podían enseñar y aprender.

Para el desarrollo de los contenidos, el maestro seguía un orden riguroso, atendiendo a la complejidad y el momento en que se realizaban a lo largo de la jornada. Quedaban para las últimas horas aquellas que se suponían menos complicadas. En este sentido, debemos resaltar la idoneidad del cuaderno como soporte para el cumplimiento de esta función, ya que todos los trabajos que el alumno realizaba en ellos cumplían con los requisitos de ordenación y secuenciación necesarios para llevar a cabo el aprendizaje de procedimientos. La labor del maestro de las "clases de pago" consistía en reforzar las materias instrumentales, imprescindibles para que el alumno fuera capaz de desenvolverse de forma autónoma.

En cuanto a la transmisión de los contenidos actitudinales, debemos destacar que existen diferencias significativas entre ambos maestros. Por un lado, el maestro del Grupo Escolar se preocupaba de impregnar cada lección de los valores y normas propias del franquismo, utilizando como recurso los dictados y creando condiciones para que los alumnos los asimilaran. De esta manera podemos confirmar que existe gran coherencia entre las intenciones educativas, los valores que transmiten los cuadernos y la fidelidad del maestro hacia las directrices del Régimen. Y es importante reiterar la coincidencia entre los textos que reproducía el alumno en sus cuadernos, con lo propuesto por la Enciclopedia. Para el desarrollo de actitudes, concluimos que el maestro tuvo en cuenta los aspectos cognitivo (consideraba necesario que los alumnos conocieran en qué consistía la actitud), afectivo (las actitudes se debían sentir interiormente) y conductual (se ponían de manifiesto con comportamientos socialmente aceptados).

Los cuadernos escolares fueron utilizados por el maestro como instrumentos de evaluación basados en los procesos elaborados por el alumno. Constituyen un reflejo de los aprendizajes esperados y las competencias adquiridas que preparaban a los niños para la vida a través del trabajo individual en el aula. En este sentido, lo que el maestro pretendía evaluar se enfocaba hacia las habilidades, capacidades y conocimientos relacionados con la práctica, sin olvidar los valores y la ideología de la época que se debían transmitir.

Como hemos señalado antes, el análisis de esta colección de cuadernos nos ha permitido realizar una aproximación a la escuela del franquismo en un contexto rural. En este sentido, queremos resaltar que un cuaderno escolar no es una fuente neutra de investigación, sino que debe concebirse como un reflejo de los procesos de enseñanza y aprendizaje protagonizados por maestro y alumno, en un tiempo histórico concreto, que nos permite apreciar la cotidianidad de la escuela en la que fue elaborado. Por ello, utilizar como fuentes primarias los cuadernos, nos exige conocer el contexto en que fueron elaborados, debemos identificar dónde tuvo lugar el hecho educativo, para así poder dar sentido a la interacción entre los sujetos que protagonizaron el proceso educativo de una escuela inmersa en una sociedad, enmarcada en una ideología y una política educativa concretas. 


\section{Referencias bibliográficas}

Apple, M. (1993). El libro de texto y la política cultural, Revista de Educación, 301, 109-126.

Álvarez Méndez, J. (1993). El alumnado. La evaluación como actividad crítica de aprendizaje, Cuadernos de Pedagogía, 219, 28-32.

Álvarez Pérez, A. (1954). Enciclopedia: Intuitiva-Sintética-Práctica. Tercer Grado. Zamora: Elma.

Badanelli Rubio, A. M. y Mahamud Angulo, K. (2007). Posibilidades y limitaciones del cuaderno escolar como material curricular. Un estudio de caso, Avances en supervisión educativa: Revista de la Asociación de Inspectores de Educación de España.

Badanelli, A. y Mahamud, K. (2011). Peeking into the Classroom of Post Civil War Spain. En S. Braster, I. Grosvenor \& M. Pozo (eds.), The Black Box of Schooling. Brussels: Peter Lang, 8198.

Benso Calvo, C. (2006). Tradición y renovación en los métodos de enseñanza. En A. Escolano BeNITO (dir.), Historia Ilustrada de la escuela en España. Dos siglos de perspectiva histórica. Madrid: Fundación Germán Sánchez Ruipérez, 403-423.

Castillo Gómez, A. y Sierra Blas, V. (2008). Mis primeros pasos. Alfabetización, escuela y usos cotidianos de la escritura (siglos XIX y XX). Gijón: Ediciones Trea.

De Iriarte, T. (1893). Fábulas Literarias. Madrid: Editorial Calleja.

Del Pozo Andrés, M. M. y Ramos Zamora, S. (2001). El cuaderno de clase como instrumento de acreditación de saberes escolares y control de la labor docente. En La acreditación de saberes y competencias. Perspectiva histórica. XI Coloquio Nacional de Historia de la Educación. Oviedo: SEDHE, Universidad de Oviedo.

Del Pozo Andrés, M. M. y Ramos Zamora, S. (2003). Los cuadernos escolares como representaciones simbólicas de la cultura escrita escolar. En Etnohistoria de la escuela. XII Coloquio Nacional de Historia de la Educación. Burgos: Universidad de Burgos, SEDHE.

Escolano Benito, A. (2002). La educación durante el franquismo. En A. Escolano Benito, La Educación en la España contemporánea. Políticas educativas, escolarización y culturas pedagógicas. Madrid: Biblioteca Nueva, 159-218.

García Hoz, V. (dir.) (1964). Diccionario de pedagogía Labor. Barcelona: Labor.

Gervilla Castillo, E. (2006). La escuela del nacional-catolicismo. Cercanía cronológica y distanciamiento axiológico, Bordón, 58 (4-5), 537-550.

Gronlund, N. (1973). Medición y evaluación en la enseñanza. México: Pax-México.

Gvirtz, S. (1999). El discurso escolar a través de los cuadernos de clase. Buenos Aires: Editorial Universitaria de Buenos Aires.

JACKSON, P. (1975). La vida en las aulas. Madrid: Ediciones Marova.

Jorba, J. y SAnMartí, N. (2000). La función pedagógica de la evaluación. En M. Ballester y otros, Evaluación como ayuda al aprendizaje. Barcelona: Graó.

Lomas, C. (2002). La vida en las aulas. Memoria de la escuela en la literatura. Barcelona: Paidós Ibérica.

Mahamud Angulo, K. (2010). Labor docente y trabajo escolar bajo el nacionalcatolicismo franquista (1945-1959). En J. Meda, D. Montino y R. SAni, (ed.), School Exercise Books. A Complex Source for a History of the Approach to Schooling and Education in the $19^{\text {th }}$ and $20^{\text {th }}$ Centuries. Florence: Edizioni Polistampa (2), 747-767.

Mayordomo Pérez, A. (1997). Nacional-catolicismo, tecnocracia y educación en la España del franquismo (1939-1975). En A. Escolano Benito y R. Fernández Losada (eds.), Los caminos hacia la modernidad educativa en España y Portugal (1800-1975). Zamora: Fundación Rei Afonso Henriques, 147-174. 
NaVArRo SANDAlinas, R. (1990). La enseñanza primaria durante el primer franquismo (1936-1975).

Barcelona: Promociones y Publicaciones Universitarias.

PARCERISA ArÁn, A. (1996). Materiales curriculares. Barcelona: Graó.

Rosales López, C. (1988). Criterios para una evaluación formativa. Madrid: Narcea.

Rosales LóPez, C. (1990). Evaluar es reflexionar sobre la enseñanza. Madrid: Narcea.

SACCHETTO, P. (1986). El objeto informador. Los objetos en la escuela: entre la comunicación y el aprendizaje. Barcelona: Gedisa.

SÁncheZ-Redondo Morcillo, C. (2004). Leer en la escuela durante el franquismo. Cuenca: Ediciones

Universidad de Castilla-La Mancha.

Serrano De Haro, A. (1941). La escuela rural. Madrid: Editorial Escuela Española.

Serrano De Haro, A. (1944). Los cimientos de la obra escolar. Madrid: Escuela Española.

SHORES, E. (2007). El portafolio paso a paso. Barcelona: Graó.

STAKE, R. (1998). Investigación con estudio de casos. Madrid: Morata.

ViñaO Frago, A. (1998). Tiempos escolares, tiempos sociales. Barcelona: Ariel.

Woods, P. (1989). La escuela por dentro. Barcelona: Paidós Ibérica.

\section{Abstract}

The teacher's labour in school exercise books: a case study

INTRODUCTION. School exercise books are important documentary sources that enable the study of the methodology, teaching strategies and patterns on which each teacher relied for support. They constitute a record of his way of working in a specific space and time - in this case, in the province of Malaga during the period of national-Catholicism. The study focuses on a collection of school exercise books that serve as primary sources, framed in a specific historical and educational context. METHODS. A qualitative study has been carried out in order to establish the emergent categories that have appeared during the process of reviewing the school exercise books. In this way, it was possible to include new variables arising from the documents. This work did not start out from preconceived conceptual categories, but rather identified them as the data was reviewed in order to carry out a descriptive analysis. This analysis allows us to access the knowledge of subjective processes where our main interest is the perspective of the social actors involved. RESULTS. An analysis of school exercise books has allowed us to demonstrate the potential and limitations they have as a source for research in the History of Education, and also to deduce the organization of school times and the intervention of the teacher in the evaluation process. DISCUSSION. From these results, we can conclude that school exercise books are effective assessment tools that allow teachers to continuously evaluate the teaching process and to guide subsequent learning achievements. They also offer students the opportunity to integrate their knowledge in a positive way and allow us to recover the learning processeses associated with different school models.

Keywords: School Exercise Books, History of Education, Formative Evaluation. 
Résumé

Le travail du professeur dans les cahiers scolaires. une étude de cas

INTRODUCTION. Les cahiers scolaires sont des sources documentaires qui permettent l'étude de la méthodologie, les stratégies et les modèles d'enseignement que chaque professeur employait pour l'enseignement. Ils constituent un registre de sa manière de travailler dans un temps et un espace déterminés, dans ce cas concret, à Malaga pendant le national-catholicisme. Cette recherche utilise, en tant que source primaire, une collection de cahiers qui appartiennent à un contexte historique et éducatif bien défini. MÉTHODE. On a réalisé une étude qualitative, compte tenu de nouvelles catégories qui ont émergé dans le processus d'examen des cahiers d'école. Ainsi, on a permis l'inclusion de nouvelles variables qui pourraient fournir les documents. Il ne s'agissait pas de partir de catégories conceptuelles préalablement identifiées, mais de les énoncer tandis que les données été examinées afin de réaliser une analyse descriptive. Cette analyse nous permet d'accéder à la connaissance des processus subjectifs où la perspective des acteurs sociaux impliqués est le plus important. RÉSULTATS. Analyser ces cahiers d'école nous a permis de démontrer leurs possibilités et leurs limites en tant que source pour l'histoire de l'éducation, pour connaître l'organisation du temps scolaire et l'intervention de l'enseignant dans le processus d'évaluation. DISCUSSION. En partant de ces résultats, on peut conclure que les cahiers d'école sont des moyens d'évaluation qui permettent aux enseignants d'évaluer les processus d'enseignement et orienter les suivants apprentissages. Également, ils offrent aux étudiants la possibilité d'intégrer leurs connaissances d'une manière positive et nous permettent de récupérer les processus d'apprentissage associés aux différents modèles de l'école.

Mots clés: Cahiers d'école, Histoire de l'Éducation, Évaluation formative.

\section{Perfil profesional de las autoras}

\section{Carmen Sanchidrián Blanco}

Catedrática de Historia de la Educación y directora del Departamento de Teoría e Historia de la Educación de la UMA. Sus líneas de investigación incluyen la cultura material de la escuela, la historia de la escuela y de la infancia, y el uso de la iconografía en la historia de la educación. En los últimos años ha participado en proyectos relacionados con estos temas.

Correo electrónico de contacto: sanchidrian@uma.es

\section{Bárbara Arias Gómez}

Profesora de Educación Secundaria de la Consejería de Educación de la Junta de Andalucía. Ha realizado el Máster en Cambio Social y Profesiones Educativas. Forma parte del grupo de investigación HUM-387 de Historia de la Educación y está realizando la tesis doctoral sobre el estudio histórico de cuadernos escolares, tema en el que ya tiene varias publicaciones.

Correo electrónico de contacto: barbararias@gmail.com 
